\title{
Tyrosine kinase inhibition produces specific alterations in axon guidance in
}

\section{the grasshopper embryo}

\author{
Kaushiki P. Menon and Kai Zinn* \\ Division of Biology, California Institute of Technology, Pasadena, CA 91125, USA \\ *Author for correspondence (e-mail: zinnk@cco.caltech.edu) \\ Accepted 24 July; published on WWW 14 September 1998
}

\section{SUMMARY}

Tyrosine kinase signaling pathways are essential for process outgrowth and guidance during nervous system development. We have examined the roles of tyrosine kinase activity in programming growth cone guidance decisions in an intact nervous system in which neurons can be individually identified. We applied the tyrosine kinase inhibitors herbimycin $A$ and genistein to whole $40 \%$ grasshopper embryos placed in medium, or injected the inhibitors into intact grasshopper eggs. Both inhibitors caused interneuronal axons that normally would grow along the longitudinal connectives to instead leave the central nervous system (CNS) within the segmental nerve root and grow out toward the body wall muscles. In addition, herbimycin A produced pathfinding errors in which many longitudinal axons crossed the CNS midline. To study how this drug affected guidance decisions made by individual growth cones, we dye-filled the pCC interneuron, which normally extends an axon anteriorly along the ipsilateral longitudinal connective. In the presence of herbimycin $\mathrm{A}$, the pCC growth cone was redirected across the anterior commissure. These phenotypes suggest that tyrosine kinase inhibition blocks a signaling mechanism that repels the growth cones of longitudinal connective neurons and prevents them from crossing the midline.

Key words: Tyrosine kinase, Grasshopper, Midline, Neural development, Growth cone

\section{INTRODUCTION}

Neuronal processes in developing embryos extend along defined pathways to find their appropriate synaptic partners. Growth cones, which form the leading edges of processes, sample the environment and make pathfinding decisions. Contacts between surface proteins on growth cones and guidance cues on surrounding cells and in the extracellular matrix affect signal transduction cascades in the growth cones. These signaling events change the growth cone's cytoskeleton, altering its morphology and direction of movement. The growth cone also transmits information about its environment back to the cell body in order to reprogram gene expression in preparation for the next pathway decision (Von Bernhardi and Bastiani, 1995).

Pathways that control tyrosine phosphorylation are important components of growth cone signaling, and mutations in genes encoding tyrosine kinases (TKs) and phosphatases have selective effects on axon guidance during embryogenesis. In mice, mutations in the EphB2 and EphB3 genes, which encode Eph receptor TKs, affect formation of the anterior commissure and the corpus callosum (Henkemeyer et al., 1996; Orioli et al., 1996). In Drosophila, mutations in the Derailed receptor TK gene alter guidance of a subset of interneuronal axons (Callahan et al., 1995; Speicher et al., 1998). Mutations in three Drosophila receptor tyrosine phosphatase (RPTP) genes affect specific guidance decisions made by motor axons (Desai et al., 1996, 1997; Krueger et al., 1996).

Src family nonreceptor TKs are highly expressed in growth cones and their filopodial tips, which are actively involved in exploring the environment (Bixby and Jhabvala, 1993). These cytoplasmic TKs are involved in transmitting signals from cell adhesion molecules (CAMs) that affect neurite outgrowth. While null mutants for the Src, Fyn and Yes TKs do not display dramatic neurological abnormalities, cultured cerebellar granule neurons from these mutant mice have altered neurite outgrowth properties (reviewed by Lowell and Soriano, 1996).

The roles of TKs in controlling process outgrowth have also been addressed by pharmacological experiments in which drugs that inhibit TK activity are added to cultured neurons. In studies on dissociated neurons, TK inhibition sometimes reduced and sometimes promoted outgrowth, depending on the type of neuron used (Bixby and Jhabvala, 1992; Doherty et al., 1994; Miller et al., 1993; Worley and Holt, 1996). Herbimycin A (HA), which has some specificity for Src-family TKs, reduced the rate of outgrowth in the Xenopus exposed brain preparation, in which retinal axons navigate to their tectal targets in the context of an intact brain (Worley and Holt, 1996).

TK inhibitors have many biochemical effects in cultured cells. In one recent study, HA and a more broad-spectrum TK inhibitor, genistein (GNS), were found to block recruitment of 
a large set of signaling molecules to focal adhesion complexes (Miyamoto et al., 1995). Signaling proteins that required TK activity for assembly into complexes included Src-family kinases, as well as Rac and RhoA, which are Ras-superfamily GTPases that regulate cytoskeletal dynamics. Rac is required for sensory and motor axon guidance in Drosophila (Kaufmann et al., 1998; Luo et al., 1994). These and other data suggest that TKs might regulate cytoskeletal changes in growth cones in part via alterations in localization or activity of Raclike GTPases.

In order to directly address TK function in programming axon guidance decisions, we used HA and GNS to inhibit TK activity during development of the grasshopper CNS. The grasshopper nervous system has large, individually identifiable neurons, and it can develop for up to 3 days (10-15\% of normal development) in a whole embryo placed in nutrient medium (Myers and Bastiani, 1993). During this time, neuroblasts generate normal neuronal and glial lineages (Condron and Zinn, 1994), and neurons differentiate and extend axons along the same pathways that are used in vivo (Condron and Zinn, 1994; Myers and Bastiani, 1993). Here we show that TK inhibition produces specific and reproducible alterations in $\mathrm{CNS}$ axon guidance. HA causes growth cones to turn and cross the midline of the embryonic CNS instead of growing longitudinally, and HA and GNS both reroute interneuronal growth cones into pathways that exit the CNS.

\section{MATERIALS AND METHODS}

\section{Embryo cultures, antibody staining and cell labeling}

Embryos were staged according to criteria established by Bentley et al. (1979). 39-40\% embryos were cultured in media containing $0.08 \%$ DMSO (control) or $20 \mu \mathrm{M}$ Herbimycin A (HA) (RBI) in DMSO (same final DMSO concentration as for controls) for 20-24 hours, together with a cohort embryo (Myers and Bastiani, 1993). Genistein (GNS) (RBI) (also in DMSO) was used at a concentration of $300 \mu \mathrm{M}$. After culturing, embryos were fixed and stained with antibodies (Patel et al., 1989). HA and GNS produced no phenotypes at less than $5 \mu \mathrm{M}$ and $250 \mu \mathrm{M}$, respectively. Tyrphostin B42 produced axonal phenotypes identical to GNS. Other tyrosine kinase inhibitors that did not produce any effects were lavendustin A, lavendustin C and CGPDAPH.

Anti-PY polyclonal antibody (Zymed) was used at 1:700; anti-Syt polyclonal antibody (Littleton et al., 1993) was used at 1:900; antiLY antibody (Molecular Probes) was used at 1:1000. mAb 8B7 (recognizes a cytoskeletal component) supernatant was used at 1:7.5, $8 \mathrm{C} 6$ (anti-FasII) was used at 1:50, and 2B2 ascites was used at 1:500. These mAbs were provided by M. Bastiani, (University of Utah) (Bastiani et al., 1987; Seaver et al., 1991). Embryos were photographed on a Zeiss Axioplan microscope, and photographs were scanned and compiled using Adobe Photoshop.

For DiI and LY injections, cultured embryos were prefixed for 510 minutes (Sink and Whitington, 1991; Whitington, 1989). To gain access to the SN root, ventral muscles had to be removed with a glass needle before DiI labelling. Images were taken on a Zeiss confocal microscope.

\section{In vivo egg injections}

The method for in vivo injections was based on fly embryo injection protocols and was similar to that described by Petrey et al. (1989). Needles were filled with a solution consisting of $12.5 \mathrm{mM} \mathrm{HA}$ and food dye in $50 \% \mathrm{DMSO} / 40 \%$ ethanol/10\% aqueous solution. Control solution was the same, but without HA. The solution was injected into the posterior portion of the egg near the embryo, until color was observed in the egg. Injected eggs were incubated for 24 hours before embryos were fixed for mAb $2 \mathrm{~B} 2$ immunohistochemistry or LY injections. The amounts of control and HA solutions injected were matched using the food dye as a color indicator. The final concentration of HA in the egg was approximately $10 \mu \mathrm{M}$, assuming that the dye solution diffuses through half of the volume of the egg within 24 hours.

\section{Electron microscopy}

Samples were prepared for EM analysis as described by Bastiani et al. (1984). For EM analysis of the nerve roots, the body wall on one side was removed. Stained grids containing gold sections were examined with a Phillips 201 transmission electron microscope. To determine the number of axons in the $\mathrm{SN}$ and ISN roots, semiserial sections from drug-treated and control embryos were examined in order to find cross-sections of the roots that were as close as possible to the connective (thus ensuring that they contained profiles of all axons in the root) but did not contain any profiles of longitudinal connective axons. These cross-sections were photographed at $7100 \times$ magnification, and axons were counted in montages of the high-power electron micrographs. Statistical analysis of axon profile numbers was done using Student's $t$-test.

\section{Immunoblotting}

Lysates were made from the dissected CNS of 10 embryos placed into culture medium for 16 hours by homogenizing with a pipette tip in lysis buffer (300 mM NaCl, $50 \mathrm{mM}$ Tris- $\mathrm{HCl} \mathrm{pH}$ 7.5, 0.5\% Triton X100 ), plus $100 \mu \mathrm{M}$ PMSF, $2 \mu \mathrm{g} / \mu \mathrm{l}$ leupeptin and $1 \mathrm{mM}$ benzamide. Samples were resolved on a $6 \%$ polyacrylamide gel and transferred to PVDF filters (Biorad). The filters were blocked in 3\% BSA/0.2\% Tween-20/1× TBST (25 mM Tris-HCl pH 7.4, $137 \mathrm{mM} \mathrm{NaCl}, 0.2 \%$ Tween-20), then incubated in anti-PY mAb 4G10 (1:2000 dilution; Upstate Biotechnology). This was followed by incubation in goat antimouse HRP-labelled secondary antibody (1:2000 dilution; Jackson Immunoresearch) diluted in 5\% Blotto/0/2\% Tween-20/1× TBST and processed for chemiluminescence using the ECl-Plus kit (Amersham).

\section{RESULTS}

\section{Tyrosine kinase inhibition affects development of the grasshopper CNS}

Phosphotyrosine (PY) is abundant in vertebrate nervous systems (Bixby and Jhabvala, 1993). To determine if PY is similarly enriched in the insect nervous system, we stained developing grasshopper (Schistocerca americana) embryos with anti-PY antiserum. We found that CNS axons contained high levels of PY. At $42 \%$ of embryonic development, PY was especially abundant in the commissures, in the neuropilar region at the junctions between the commissures and connectives, and in the nerve roots in which motor axons leave the CNS (Fig. 1).

The spatially regulated pattern of PY localization suggests that signaling via control of tyrosine phosphorylation might be important for guidance of grasshopper commissural and motor axons. To examine this, we used monoclonal antibodies (mAbs) that stain commissural axons (Seaver et al., 1991) (see Fig. 4). To visualize motor axons, we used polyclonal antibodies against Drosophila synaptotagmin (Syt) (Perin et al., 1991), which we found to selectively label CNS and motor pathways in the grasshopper. Fig. 2A shows anti-Syt staining of abdominal segment $\mathrm{A} 3$ at $50 \%$ of development, and the schematic diagram of Fig. 2B depicts the motor axon and 


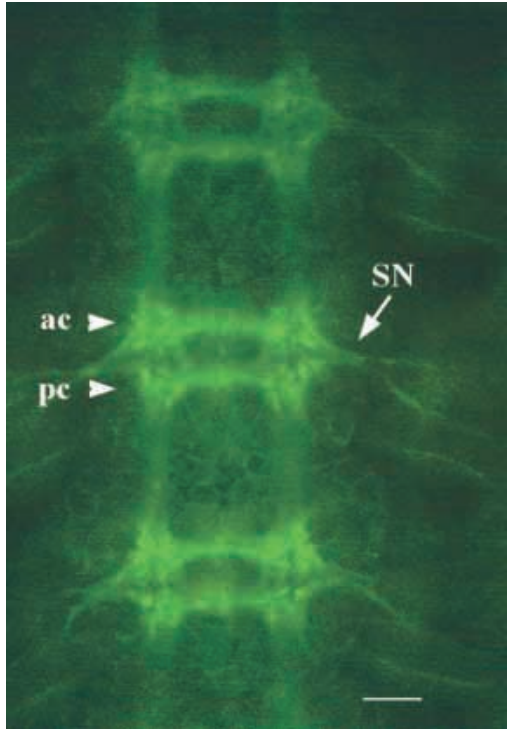

Fig. 1. Anti-phosphotyrosine staining of grasshopper ventral nerve cord. $42 \%$ embryos were stained with anti-PY polyclonal antibody, followed by fluorescent secondary antibody. Abdominal segments $\mathrm{A} 2$ to $\mathrm{A} 4$ are shown here. ISN is in a different focal plane than the one shown here. (ac) anterior commissure; (pc) posterior commissure; (SN) segmental nerve. Scale bar, $10 \mu \mathrm{m}$.

muscle patterns in an abdominal segment of approximately this age (Xie et al., 1992). A complete characterization of the development of the motor axon pattern will be presented elsewhere.

The grasshopper CNS develops in a normal manner in whole embryos incubated in a nutrient medium (Condron and Zinn, 1994; Myers and Bastiani, 1993). In our experiments, we placed 39-40\% embryos in medium and monitored their development by staining with anti-Syt. Extensive CNS development occurred within a 24 hour period (compare Fig. 3A and B). The overall pattern of anti-Syt stained axons after 24 hours in medium was indistinguishable from the in vivo pattern at the corresponding time of development. This is about 45\%, since grasshopper embryos develop at a rate of $5 \%$ per day, hatching after 20 days. Muscle fibers also developed normally, as assayed by rhodaminephalloidin staining (data not shown).

To address the roles of TK activity during CNS axonogenesis, we screened several TK inhibitors at various concentrations for effects on development of grasshopper nervous system (see Materials and Methods). Here we report data for two inhibitors, Herbimycin A (HA) and genistein (GNS), which altered axon guidance but did not adversely affect cell survival. We performed most experiments at $40 \%$ of development, so that effects on axonogenesis could be examined without affecting neurogenesis.

Embryos treated with $20 \mu \mathrm{M}$ HA for 20-24 hours developed differently from control embryos. Control embryos had dark anti-Syt staining in the connectives, and weak staining in the commissures (Fig. 3B). Embryos treated with HA had broader, more darkly stained commissures and thinner connectives (Fig. 3C). Their CNS scaffolds were narrower in the commissural region, possibly due to increased tension across the midline exerted by the presence of larger numbers of commissural axons (reviewed by van Essen, 1997, see Condron and Zinn, 1997). HA-treated embryos also had thicker $\mathrm{SN}$ roots than control embryos (see Fig. 6). Embryos treated with $300 \mu \mathrm{M}$ GNS did not develop the commissural phenotype and their commissures were quite similar to those of control embryos (Fig. 3D). Like HA-treated embryos, however, they had SN roots that were much thicker than normal (see Fig. 6). HA and GNS did not affect the thickness of ISN roots (Fig. 3), and ISN secondary branches onto ventral and dorsal muscles formed in a normal manner (data not shown).

\section{Specific effects of herbimycin A on commissural and longitudinal CNS pathways}

The broadened and darker commissures observed in HAtreated embryos stained with anti-Syt could be due to the presence of larger numbers of commissural axons, or to overexpression of Syt in the commissures. To further evaluate the effects of HA and GNS on commissural and longitudinal pathways, we stained control, HA-treated and GNS-treated embryos with other marker antibodies. mAb $2 \mathrm{~B} 2$ recognizes an epitope secreted from axons but localized near the axonal scaffold (Seaver et al., 1991). Staining with 2B2 mAb clearly revealed the broadening of the commissures caused by HA treatment (Fig. 4A,B). The anterior commissure in particular was much thicker in HA-treated embryos.

The Fasciclin II (FasII) CAM is expressed at high levels on
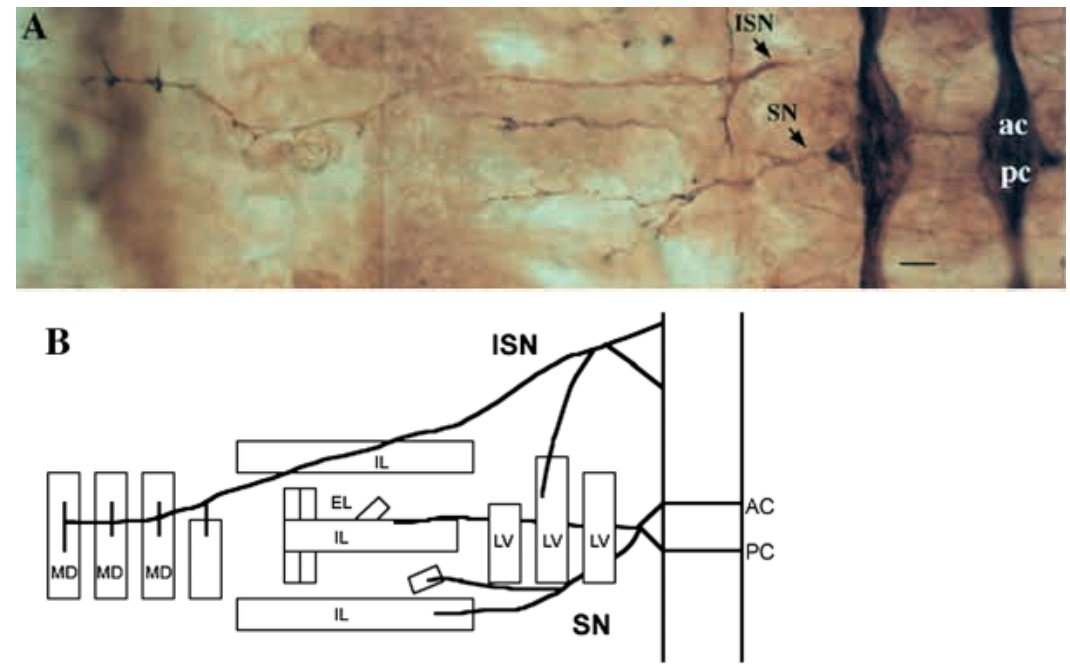

Fig. 2. Anatomy of grasshopper motor nerves. (A) Embryos at 50\% development were stained with anti-Syt polyclonal antibody. A composite picture of bright-field photographs taken at different focal planes of an A3 segment is shown. Scale bar: $7.1 \mu \mathrm{m}$. (B) Schematic drawing of motor pathways and muscle fibers in an abdominal segment. The diagram was derived from confocal images of $50 \%$ embryos double-stained with anti-Syt and rhodamine-phalloidin. The nomenclature for the muscles is from Xie et al., 1992. Not all muscles are shown. ac, anterior commissure; pc, posterior commissure; SN, segmental nerve; ISN, intersegmental nerve; MD, median dorsal muscle; IL, internal lateral muscle; EL, external lateral muscle; LV, lateral ventral muscle. 
Fig. 3. Effects of tyrosine kinase inhibitors on CNS axonogenesis. Segments A3-A5 are shown. (A) Control $39 \%$ embryo stained with anti-Syt at the beginning of the culture period.

(B-D) Embryos from the same clutch stained with anti-Syt after 24 hours of culture, in control medium plus DMSO (final DMSO concentration equal to that in drug-containing media) (B), HAcontaining medium (C), or GNScontaining medium (D). Embryos were photographed in bright field. ac, anterior commissure; pc, posterior commissure. Scale bar $10 \mu \mathrm{m}$.

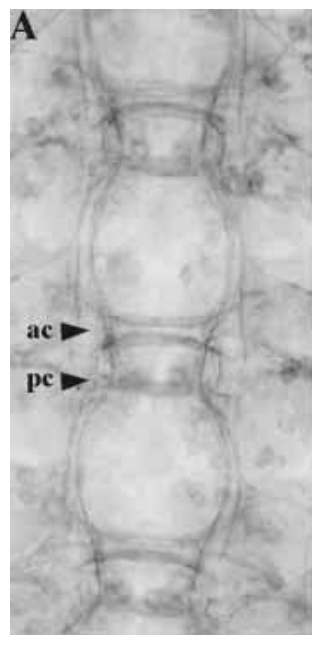

three longitudinal bundles, and at lower levels on other commissural and longitudinal tracts (Bastiani et al., 1987) (Fig. 4D). In both HA- and GNS-treated embryos, there was an apparent decrease in the number of axons in the Fas II bundles (Fig. 4E,F). The thinning of these connective bundles was more dramatic in HA-treated than in GNS-treated embryos.

To determine whether the guidance changes that we saw in cultured whole embryos could also be produced in vivo, we injected HA solutions directly into grasshopper eggs at $36 \%$ or $40 \%$ of development, and dissected and stained the embryos 24 hours later. Younger embryos (35-36\%) exhibited morphological alterations when cultured in the presence of TK inhibitors. However, these morphological changes did not occur when HA was injected into intact eggs at this age. Embryos from eggs injected with control solutions developed normally. Embryos from eggs injected near the posterior end with $\mathrm{HA}$, however, exhibited the same alterations in mAb 2B2 staining patterns (Fig. 4C) as did embryos cultured in the presence of HA (Fig. 4B).

We observed no changes in the neuronal expression patterns of the Even-skipped (Eve) and Engrailed transcription factors in cultured embryos treated with HA. The patterns of muscles and of sensory neurons and their axons were normal. Midline glia also developed normally, as assayed by staining for the glial surface marker Annulin (Bastiani et al., 1992) (data not shown). These data suggest that, at the stage of development used for our experiments, the effects of HA were relatively specific to CNS axons.

In summary, the antibody staining patterns indicate that additional axons were added to the commissures in embryos treated with HA in culture or in vivo. There were also fewer axons in the longitudinal connectives of these embryos. These results suggest that axons that normally extend along longitudinal pathways were diverted across the midline as a consequence of TK inhibition.

\section{The pCC neuron extends a collateral axon branch across the midline in herbimycin A-treated embryos} pCC and aCC are sibling neurons that express Eve (Patel et al., 1992) and their cell bodies are located just posterior to the posterior commissure. pCC is an interneuron whose axon extends anteriorly along the longitudinal connective for several segments, following a pathway established by the posteriorly
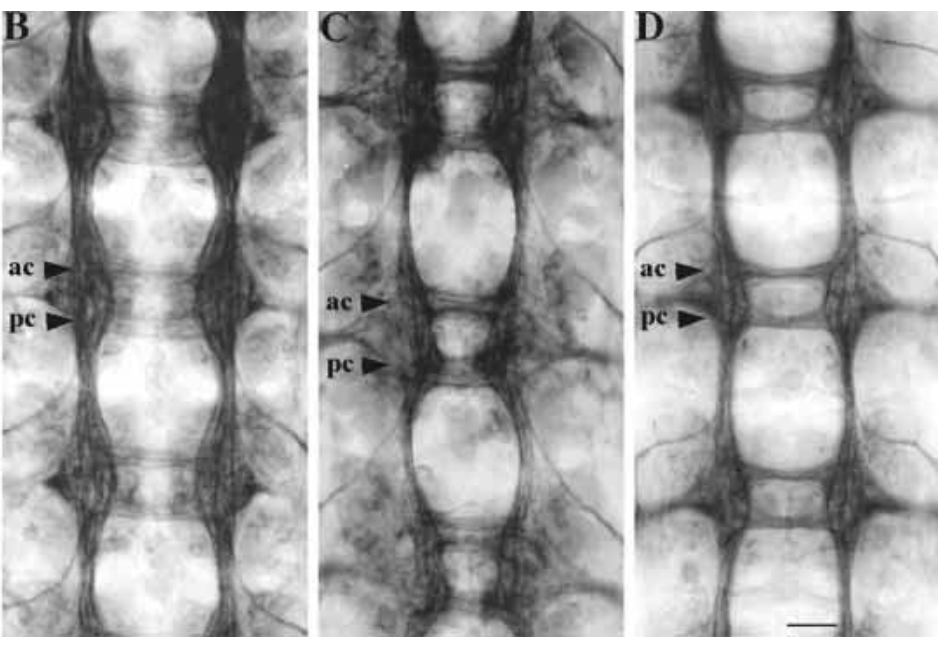

growing axons of the MP1 and dMP2 neurons (Bastiani et al., 1986) (Fig. 5A). aCC is a motor neuron that extends an axon posteriorly in the longitudinal connective. The aCC axon then turns into the ISN root and leaves the CNS, eventually innervating one of the dorsal body wall muscles. The perturbation of longitudinal and commissural pathways by HA, and the lack of an apparent effect on the ISN, suggested to us that pCC axon guidance might be altered by the inhibitor, while aCC might be unaffected. We investigated this by filling pCC and aCC with Lucifer Yellow (LY) dye.

As expected, in control embryos pCC axons followed the normal pathway along the ipsilateral longitudinal connective (Fig. 5A) ( $n=6$ in 3 embryos) (Bastiani et al., 1986). In HAtreated embryos, however, the pCC axon never behaved normally (Fig. 5B,C) ( $n=33$ in 11 embryos). Grasshopper CNS segments develop in an anterior-to-posterior sequence, so that development of most aspects of the CNS in a particular abdominal segment is delayed by about $1 \%$ relative to the next anterior segment. In older (more anterior) segments, in which the pCC axon had already extended past the anterior commissure of its own segment when the embryo was placed into HA-containing medium, we observed that, although the main axon followed the normal pathway, pCC also extended a collateral branch across the midline (21/25 hemisegments examined; Fig. 5B). The collateral branch followed a pathway near the posterior edge of the anterior commissure. It often turned posteriorly after crossing the commissure, following a pathway near the medial edge of the longitudinal connective. In the other four hemisegments, collateral processes that appeared thinner than axons were observed to extend across the midline. These may be pCC filopodia that were exploring a commissural pathway. Production of a collateral branch could be observed as early as 8 hours after addition of HA (data not shown).

In younger (more posterior) segments in which the pCC axon was close to the posterior edge of the anterior commissure at the beginning of the culture period, the entire pCC axon turned and extended across the midline in the anterior commissure (8/8 such segments examined; Fig. 5C). Like the collateral branch seen in older segments, the diverted pCC axon then turned posteriorly, and sometimes extended filopodia back across the posterior commissure. In the segment shown, other filopodia also extended across the posterior commissure 
Table 1. Number of axons in the SN roots of control and perturbed embryos

\begin{tabular}{|c|c|c|c|}
\hline Embryo & Segment & $\mathrm{SN}$ & ISN \\
\hline \multirow[t]{4}{*}{ Control 1} & $\mathrm{~A} 3 \mathrm{i}$ & 48 & 27 \\
\hline & A $4 \mathrm{i}$ & 45 & 29 \\
\hline & A5i & 48 & 29 \\
\hline & A6i & 44 & 28 \\
\hline \multirow[t]{6}{*}{ Control 2} & A5i & 44 & 29 \\
\hline & A6i & 44 & 31 \\
\hline & A7i & 49 & 25 \\
\hline & A5c & 48 & 27 \\
\hline & A6c & 46 & 28 \\
\hline & $\mathrm{A} 7 \mathrm{c}$ & 44 & 30 \\
\hline \multirow[t]{4}{*}{ HA 1} & A3 & 130 & 27 \\
\hline & A4 & 141 & 27 \\
\hline & A5 & 104 & 28 \\
\hline & A6 & 150 & 24 \\
\hline \multirow[t]{2}{*}{ HA 2} & A3 & 126 & 28 \\
\hline & A4 & 151 & 26 \\
\hline \multirow[t]{4}{*}{ GNS 1} & A3 & 127 & 26 \\
\hline & A4 & 128 & 20 \\
\hline & A5 & 116 & 14 \\
\hline & A6 & 107 & 17 \\
\hline
\end{tabular}

$\mathrm{SN}$, segmental nerve root; ISN, intersegmental nerve root; i, ipsilateral segments; c, contralateral segments

from the proximal portion of the axon, so the whole $\mathrm{pCC}$ axon almost formed a loop.

We also filled pCC neurons in embryos from HA-injected eggs that had been injected with HA or control solutions at $36 \%$ of development. Thus, Fig. 5E,F shows the effects of TK inhibition at an earlier stage of pCC axonogenesis. Here the control pCC (Fig. 5E) extended its axon to the posterior commissure of the next anterior segment. The pCC growth cone in the HA-treated embryo, however, stayed at a choice point at the anterior commissure of the same segment (Fig. 5F). It radiated large numbers of filopodia, most of which extended across the midline in one of the two commissures. Filopodia also extended anteriorly, apparently following the MP1/dMP2 pathway that would normally be selected by the pCC growth cone. Very few filopodia extended in directions away from the midline. These findings suggest that the growth cone in the HA-treated embryo was in the process of making the abnormal decision to grow across the midline, but also retained the ability to recognize its normal pathway. pCC growth cones in control embryos never extended filopodia across the midline in this manner.

We also noted that $\mathrm{pCC}$ axons in HA-treated embryos were much shorter than in control embryos of the same age. For example, the combined length of the main axon and collateral branch in Fig. 5B is about half of the length of the pCC axon in Fig. 5A. In Fig. 5F, the pCC axon is about one-third of the length of the control axon in Fig. 5E. These effects could be due to changes in axonal growth rates, to retraction of the main axon, and/or to an increase in the amount of time at which the growth cone pauses at choice points.

The effects of HA appeared to be specific to $\mathrm{pCC}$, because the primary aCC axon followed the normal pathway and extended for the normal length in all HA-treated segments examined (Fig. 5D) ( $\mathrm{n}=12$ in 7 embryos). In one case, a collateral branch of the aCC axon was also observed, which extended back toward the CNS from the ISN root.

\section{Tyrosine kinase inhibition increases the number of axons in the SN root}

The thickness of the SN root, but not that of the ISN root, was dramatically increased in both HA- and GNS-treated embryos stained with anti-Syt (Fig. 6B,D). The SN splits into an anterior and a posterior branch immediately upon leaving the CNS (Figs $2,6 \mathrm{~A})$. The branches were of approximately equal diameter in control embryos (Fig. 6A,C). In GNS-treated embryos, the posterior branch was much thicker than the anterior (Fig. 6D).

To determine whether the increases in SN root diameter in inhibitor-treated embryos indicated that the roots contained more axons than in control embryos, we analyzed crosssections of SN and ISN roots in control, HA-treated and GNStreated embryos using transmission electron microscopy (EM). Fig. 7A is a view of an abdominal SN root from a control embryo, with the plane of section between the point at which the root exited the CNS and that at which it split into the anterior and posterior branches (see Fig. 6 to locate the plane of EM cross-sections shown in Fig. 7). Fig. 7E is a high-power view of a portion of the same root. As is commonly seen in EM sections of grasshopper embryos (Bastiani et al., 1984), the axonal profiles are arranged in widely separated bundles. Numerous filopodia are scattered throughout the intercellular space, which presumably was filled with extracellular matrix material in vivo. Fig. 7C is a view of the much smaller ISN root, which is usually tightly apposed to a cell body.

In Fig. 7B, a cross-section of an SN root from an HA-treated embryo shows many more axonal profiles than those in the roots of control embryos. When examined at high power, however (Fig. 7F), individual profiles were similar in appearance to those in control embryos. The ISN root was apparently unaffected by HA (Fig. 7D), having approximately the same number of axonal profiles as the control ISN root (Fig. 7C).

To make a quantitative estimate of the magnitude of the increase in the number of $\mathrm{SN}$ axons produced by TK inhibitor treatment, we counted axonal profiles in high-power EM views of SN roots from 10 abdominal hemisegments of control cultured embryos, 6 hemisegments of HA-treated embryos and 4 hemisegments of a GNS-treated embryo (Table 1). The control SN roots contained 44-49 axons (46 \pm 2.05$)$, while SN roots from HA-treated embryos had 104-150 axons (133.7 \pm 17.75$)$, and those from the GNS-treated embryos had 107-128 axons (119.5 \pm 9.95$)$. These differences are highly significant $(P<0.001$ for HA, $P<0.001$ for GNS $)$.

The ISN roots had approximately the same number of axons in control and inhibitor-treated embryos (25-31 for control, $28 \pm 1.73$; $24-28$ for HA, $27 \pm 1.55$; $14-26$ for GNS, $19.25 \pm 5.12$ ). There are no statistically significant differences between these numbers for control and HA-treated embryos $(P<0.15)$ (Table 1).

These results indicate that many more axons grew into the $\mathrm{SN}$ root in HA- and GNS-treated embryos than in control embryos. Although the SN root usually appeared thicker in GNS- than in HA-treated embryos, this was not due to the presence of more axons. Rather, individual axons in GNS-treated SN roots appeared to be somewhat larger (data not shown).

\section{Midline crossing and projection into the $\mathrm{SN}$ root are independent effects}

HA had several effects on the axon scaffold, causing broadening of the commissures, narrowing of the longitudinal connectives and thickening of the SN root. GNS affected the 
Fig. 4. Effects of herbimycin A on development of commissural and connective pathways. The A4 segment is shown in all panels. Embryos were incubated for 24 hours in control medium plus DMSO (A,D), or in HA-containing medium $(\mathrm{B}, \mathrm{E})$ or in GNS-containing medium (F) for 24 hours. (C) An embryo where HA was injected into the egg and incubated for 24 hours. These embryos were stained with $\mathrm{mAb} 2 \mathrm{~B} 2$, which recognizes a secreted protein (A-C); or mAb 8C6, which recognizes the FasII CAM (D-F). Embryos were photographed using DIC optics. ac, anterior commissure; pc, posterior commissure. Asterisks in $\mathrm{E}$ and $\mathrm{F}$ indicate Fas II bundles in the longitudinal connectives that were thinner than normal or absent. Scale bar, $5 \mu \mathrm{m}$.
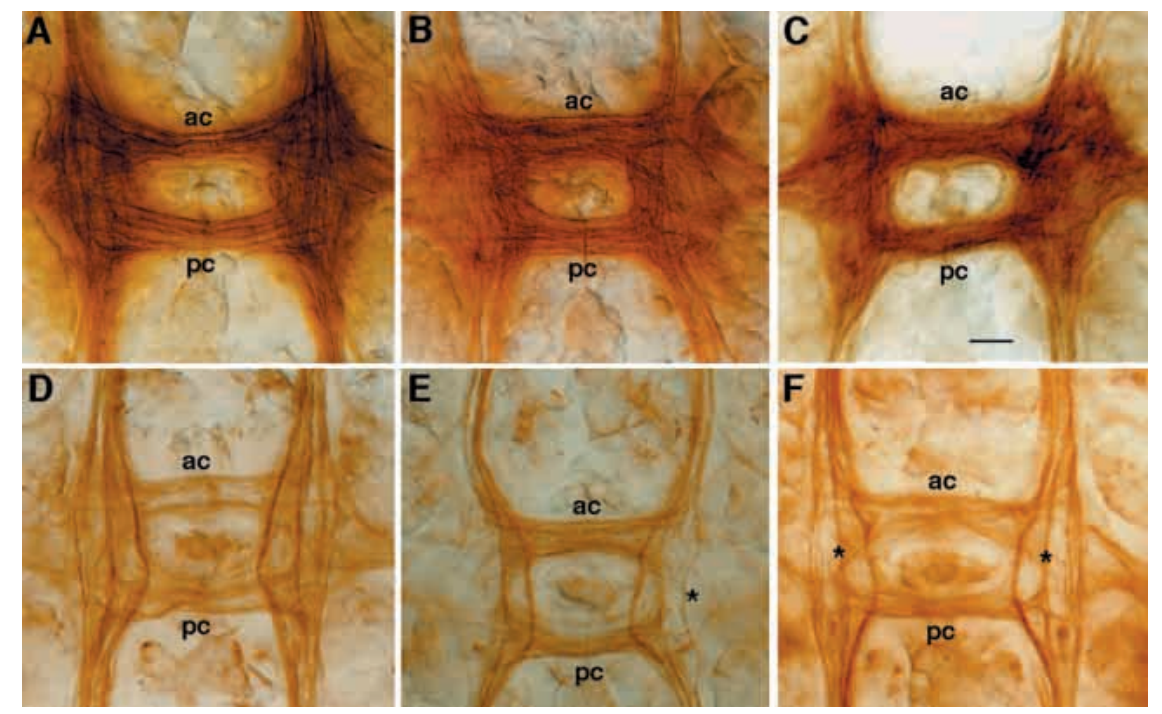

SN root and the connectives, suggesting that the commissure/connective phenotype can be produced independently of the SN root/connective phenotype. It was possible, however, that in HA-treated embryos the phenotypes were linked. This could have occurred if the rerouted longitudinal axons that crossed the midline then grew into the contralateral SN root. To evaluate this model, we backfilled SN roots in control and inhibitor-treated embryos with DiI. We expected that, by analogy to Drosophila (Landgraf et al., 1997; Sink and Whitington, 1991), SN root axons might derive from an exclusively ipsilateral population of cell bodies. If axons in the SN roots of HAtreated embryos had first crossed the midline, however, one would expect to see extensive labeling of contralateral cell bodies after backfilling.

Backfilling of $\mathrm{SN}$ roots in control embryos revealed an exclusively ipsilateral population of about 25 neurons (Fig. 8A). When $\mathrm{SN}$ roots in HA-treated embryos were backfilled, a much larger population of neurons was labeled (Fig. 8B). All but one of these were ipsilateral, however. There was no labeling of axonal tracts in the connective. When $\mathrm{SN}$ roots in GNS-treated embryos were backfilled, a similarly large population of

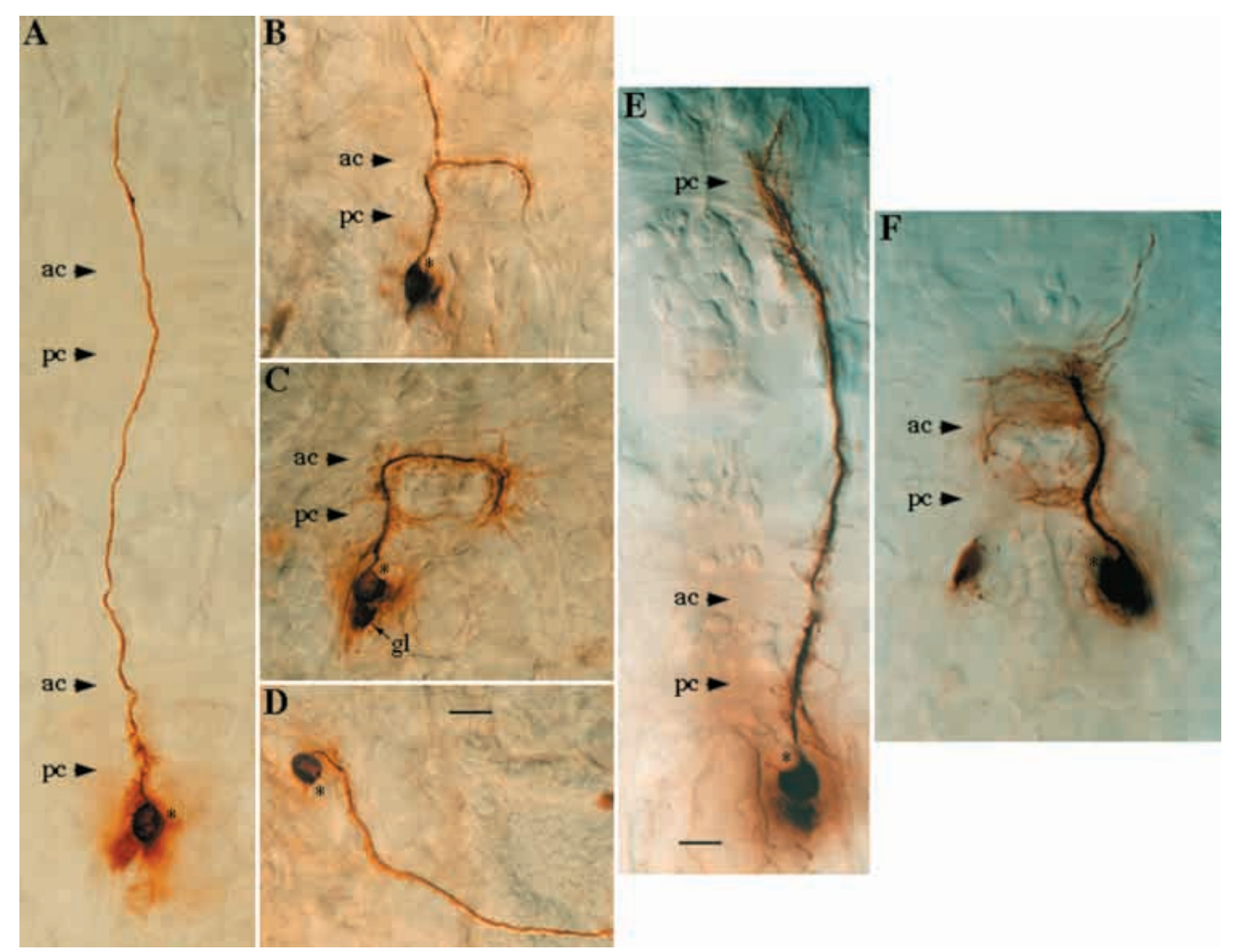

Fig. 5. Effects of herbimycin $\mathrm{A}$ on $\mathrm{pCC}$ and aCC axon guidance. $40 \%$ embryos were incubated in control medium plus DMSO (A), or in HA-containing medium (B-D) for 24 hours. (E,F) Intact eggs $(36 \%)$ were injected with control solution (E), or HA-containing solution (F). LY was injected into the pCC interneuron (A-C,E,F) or into the aCC motoneuron (D). In panels where pCC was filled, the asterisk indicates its sibling aCC; in the panel where aCC was filled, the asterisk indicates pCC (D). The arrow in $\mathrm{C}$ shows a glial cell (gl) next to pCC that was accidentally filled with LY. (A,B) Segment A4; (C,D) segment A6; (E,F) segment A1. Embryos were photographed using DIC optics. Since the pCC and aCC axons traverse multiple focal planes, images were merged in order to display the entire pathway in one panel. ac, anterior commissure; pc, posterior commissure. Scale bar, $5 \mu \mathrm{m}$. 
Fig. 6. Effects of tyrosine kinase inhibitors on the segmental nerve root. Bright-field photographs of $40 \%$ embryos incubated in control medium plus DMSO (A,C), HA-containing medium (B), or GNS-containing medium (D). After 24 hours $(\mathrm{A}, \mathrm{B})$, or 36 hours $(\mathrm{C}, \mathrm{D})$, embryos were dissected and stained with anti-Syt. The arrows indicate the segmental nerve roots of abdominal segment A4; the asterisks indicate intersegmental nerves, which is out of the focal plane shown. Vertical bars in panels A and B represent positions of the cross-sections of the $\mathrm{SN}$ root that were used for EM analysis in Fig. 7 and in Table 1. Scale bar $10 \mu \mathrm{m}$.

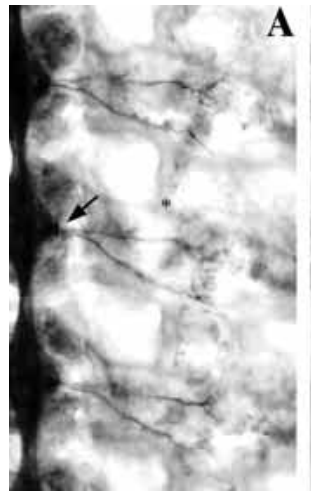

SN root axons were not collateral branches of neurons whose main axons still extended along the connective. If this were the case, backfilling the SN root would have produced extensive labeling of the ipsilateral connective. Rather, our data suggest that, in inhibitor-treated embryos, a large number of CNS
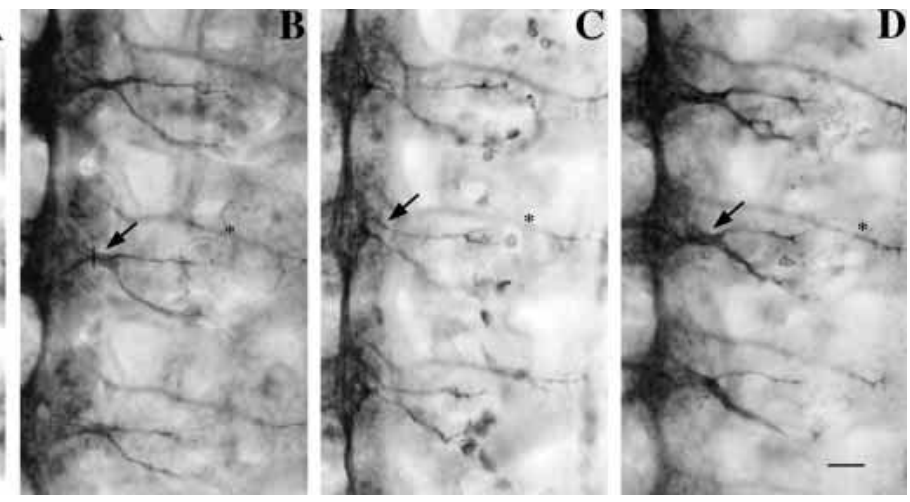

interneurons extended their primary axons into the $\mathrm{SN}$ root in preference to their normal pathway choices.

To determine approximately how many interneuronal axons were rerouted by inhibitor treatment, we first estimated the numbers of motor and sensory axons in the SN and ISN roots
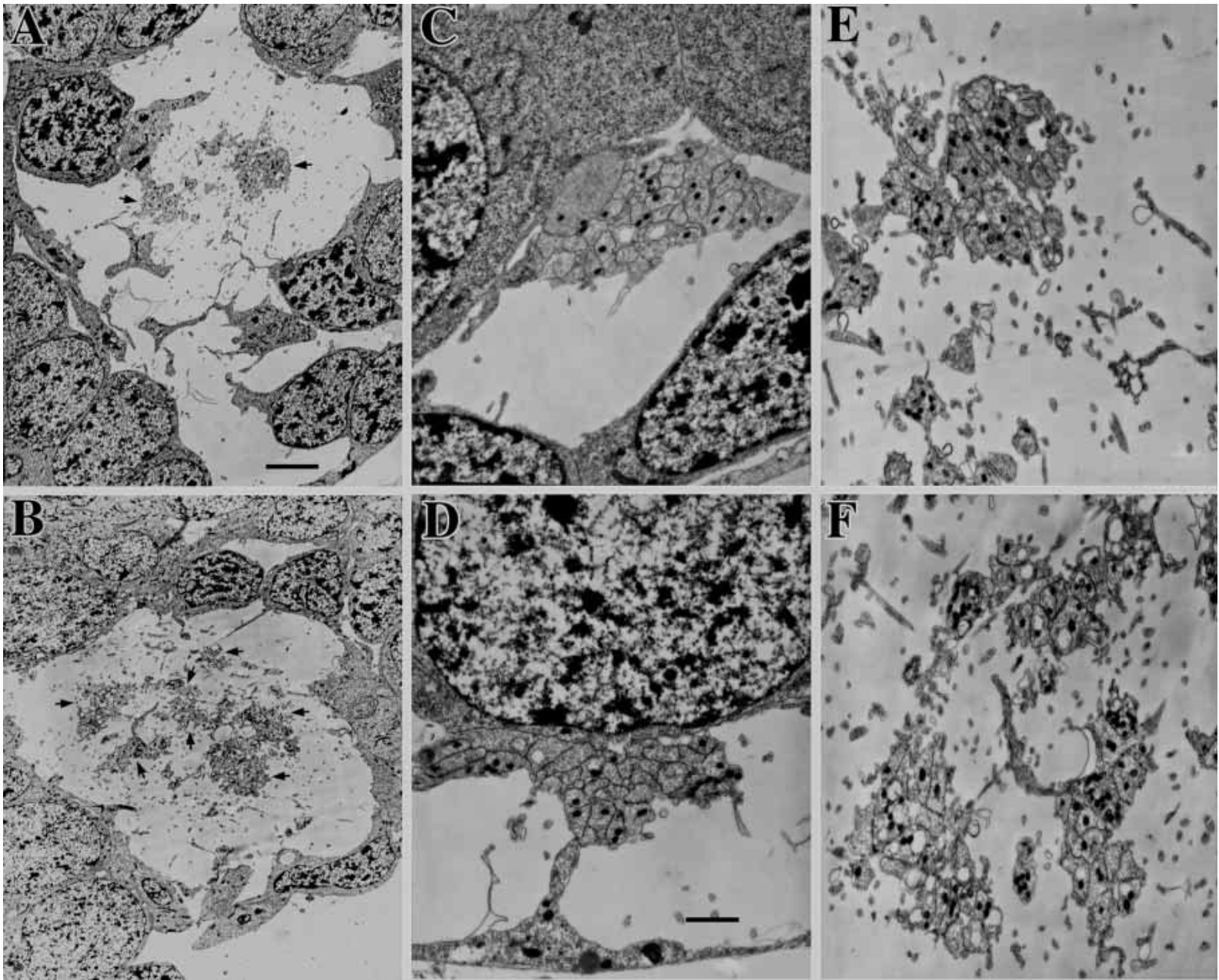

Fig. 7. Electron microscopy of segmental and intersegmental nerve roots. $40 \%$ embryos were incubated in control medium plus DMSO $(\mathrm{A}, \mathrm{C}, \mathrm{E})$, or in HA-containing medium (B,D,F) for 24 hours. In all panels, ventral is up and the basement membrane is at the bottom of the page. Segment A5 is shown in all panels. (A,B) Low power (2000× magnification) views of cross-sections through the entire SN roots. (E,F) High-power (7000× magnification) electron micrographs of portions of the roots shown in A and B. (C,D) High-power (7000× magnification) electron micrographs of the entire ISN root. Arrows in A and B indicate axon bundles in the SN root. Note that the number of axons within the white space between the cell bodies is much larger in B than in A, but that the shapes of the individual axon profiles in the two panels are similar, as seen in E,F. Scale bars: A,B, $4.8 \mu \mathrm{m}$; C-F, $1.4 \mu \mathrm{m}$. 
Fig. 8. DiI backfilling of segmental nerve roots in control and herbimycin A-treated embryos. $40 \%$ embryos were incubated in control medium plus DMSO (A), or in HA-containing medium (B). After 24 hours, SN roots in segment A4 were labelled with DiI. The cell body populations that sent axons into the SN root were imaged as confocal $z$-series projections. The large arrow indicates the approximate point in the $\mathrm{SN}$ root where DiI was applied. The arrowheads indicate the anterior and posterior branches of the SN. The small arrow in B indicates the single contralateral cell body that is labelled in HA-treated embryos. Note that the number of labeled cell bodies is much larger in B than in A. Scale bar, $15.2 \mu \mathrm{m}$.
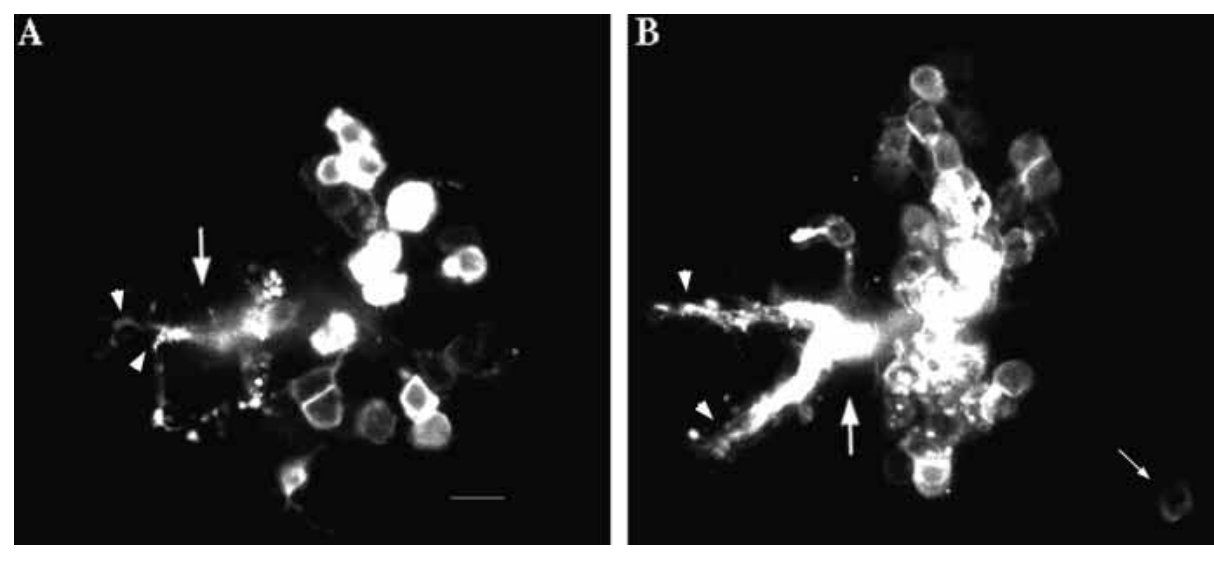

of control embryos. Previous studies of the grasshopper peripheral nervous system (PNS) (Meier et al., 1991) indicate that the SN and ISN roots each contain about 19 sensory axons. Subtraction of these numbers from total counts of axonal profiles from our EM sections then shows that the SN and ISN roots contain approximately 27 and 9 motor axons, respectively. These numbers are consistent with the number of CNS neuronal cell bodies that were labeled in our backfilling experiments (Fig. 8A and data not shown).

We then assumed that the numbers of sensory axons in the SN and ISN were the same in control and inhibitor-treated embryos. This assumption was based on the observation that the DiI backfilling experiments showed an identical pattern of labeling of sensory cell bodies in control and inhibitor-treated embryos, indicating that the same numbers of sensory neurons sent axons into the CNS through the $\mathrm{SN}$ and ISN roots. mAb 8B7, a cytoskeletal marker stains a sensory bundle that enters the CNS via the ISN root but is separated from the motor bundle in the periphery. There was no alteration in the thickness of this bundle in HA-treated embryos (data not shown).

Our data thus indicate that the number of CNS axons in the $\mathrm{SN}$ root was increased from 27 in control embryos to about 115 in HA-treated embryos. Therefore, assuming that the SN root motor axons still follow their normal pathways, in each hemisegment about 88 CNS interneurons sent their axons into the SN root as a consequence of HA treatment. This is about one-third of the total number of neurons in a hemisegment at this stage.

\section{Biochemical effects of tyrosine kinase inhibition in the grasshopper CNS}

To examine alterations in the levels of PY in specific CNS proteins in response to TK inhibitor treatment, we made lysates from the dissected CNS of control, HA- and GNS-treated embryos, fractionated equal amounts of the lysates on SDSPAGE gels and analyzed these gels by immunoblotting with an anti-PY mAb (Fig. 9). This analysis showed that the intensities of some immunoreactive bands were altered by treatment with the drugs. A prominent band at $46 \mathrm{kDa}$, which we identified as $\beta$-tubulin by immunoblotting with anti-tubulin antibodies (data not shown), was reduced in intensity by HA treatment. GNS also reduced tubulin phosphorylation, but to a lesser extent. These results indicate that an identified protein, tubulin, is hypophosphorylated in the CNS in response to the inhibitors.
Two bands at about 164 and $170 \mathrm{kDa}$ were decreased in intensity in response to HA, but less strongly affected by GNS. Bands at 103 and $117 \mathrm{kDa}$ were increased in intensity in response to HA. The intensities of many other bands were unchanged by HA or GNS. When immunoblots were done with anti-PY mAb preincubated with O-phospho-L-tyrosine, all of the bands disappeared, indicating that the anti-PY mAb was recognizing only tyrosine-phosphorylated proteins.

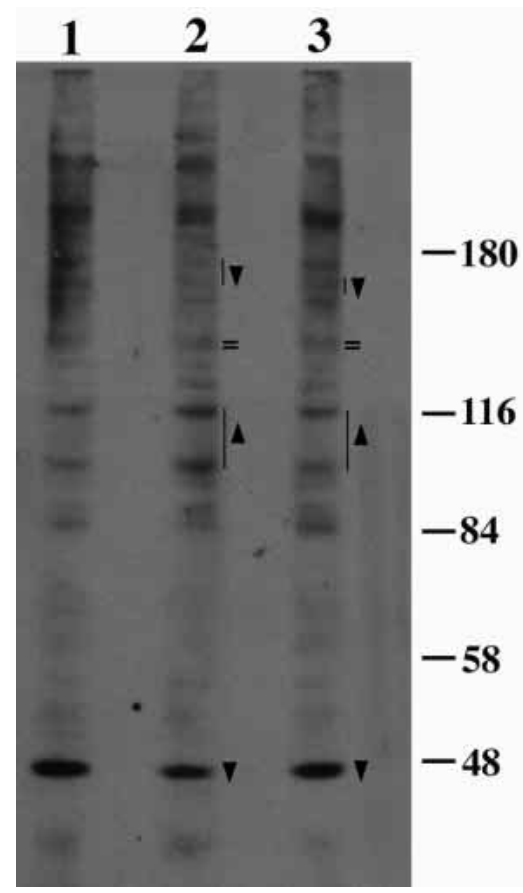

Fig. 9. Immunoblot analysis of the effects of tyrosine kinase inhibitors on tyrosine phosphorylation of CNS proteins. $40 \%$ embryos were incubated in control medium plus DMSO (lane 1), HA-containing medium (lane 2), or GNS-containing medium (lane 3) for 16 hours Markers (in $\mathrm{kDa}$ ) indicated to the right. The downward-pointing arrowheads and lines indicate bands whose intensity is decreased in response to both inhibitors. The major band below the $48 \mathrm{kDa}$ marker is $\beta$-tubulin. Upward-pointing arrowheads and lines indicate bands whose intensity is increased in response to both inhibitors. Equal signs indicate a band that has the same intensity in all lanes. 


\section{DISCUSSION}

In this paper, we examined the roles played by tyrosine phosphorylation during axonogenesis in the grasshopper embryo. We found that the tyrosine kinase inhibitors HA and GNS produced specific axonal pathfinding errors in the CNS. Both inhibitors caused interneuronal axons that normally would follow pathways along the longitudinal connectives to instead leave the CNS within the SN root (Figs 4,6-8). HA also produced pathfinding errors in which longitudinal axons cross the midline (Figs 3-5). In vertebrates, the effects of HA on axon outgrowth in an intact system have been examined using the Xenopus exposed brain preparation. Retinal axon extension toward the optic tectum was slowed when HA was applied (Worley and Holt, 1996). In contrast with our results in the grasshopper CNS, however, no pathfinding errors were observed as a consequence of TK inhibition in these experiments.

To examine the midline crossing phenotype produced by HA at the level of individual axon trajectories, we dye-filled the pCC interneuron. pCC normally extends its growth cone anteriorly within the ipsilateral longitudinal connective, following the MP1/dMP2 pathway (Bastiani et al., 1986) (Fig. 5A). The pCC axon always behaved abnormally in HA-treated embryos. Either a collateral branch (Fig. 5B) or the entire primary axon (Fig. 5C) of pCC extended across the midline. The similarity between the pathways taken by the collateral and the primary growth cone suggests that the primary growth cone resembles the abnormal collateral in its pathfinding behaviour. In both cases, the rerouted collateral or primary axon usually turned posteriorly in the connective after crossing the midline, following a pathway near the medial edge of the connective. pCC navigates anteriorly along a bundle at approximately this position, so this pathway choice could indicate that the rerouted axon fasciculated with the contralateral pCC. Finally, the axon then sometimes turned back across the midline, following a pathway within the posterior commissure. In the segment of Fig. $5 \mathrm{C}$, the pCC growth cone curved back toward the midline and sent filopodia across the posterior commissure, while other filopodia extended into this commissure from the ipsilateral side as collaterals off the primary axon. Here the entire pCC trajectory appears to be forming a closed loop. We do not know whether other axons that were rerouted across the commissures also followed looping trajectories. The pattern of staining with the 2B2 mAb seen for HA-treated embryos, however, suggests that other interneuronal axons may also follow looping pathways (Fig. 4B)

When HA treatment was initiated earlier (by in vivo injection), so that the pCC growth cone had only extended a short distance within its own segment before exposure to drug, we observed that the growth cone was still at a choice point at the anterior commissure when the embryo was dissected 24 hours later. The pCC growth cone normally pauses here for about 5 hours, at the anterior extremity of the MP1 neuron of its own segment, until the MP1/dMP2 axons from the next anterior segment reach it (Bastiani et al., 1986). It then resumes extension anteriorly along the MP1/dMP2 pathway. The pCC growth cone in Fig. 5F stalled at or near this choice point and extended many long filopodia. These emanated primarily in two directions: across the midline and anteriorly along the
MP1/dMP2 pathway. This suggests that, in the presence of HA, the pCC growth cone still recognized its normal pathway, but also had an affinity for commissural pathways. In contrast, if the MP1/dMP2 neurons were ablated, the pCC growth cone usually stalled at this choice point and did not extend filopodia across the midline (Bastiani et al., 1986). If it continued to extend, it either made a U-turn or grew out of the CNS within the ISN root. These results suggest that HA did not block recognition of the MP1/dMP2 pathway, but rather uncovered the presence of a signal that redirected the pCC growth cone across the midline.

The observation that many extra axons crossed the commissures in HA-treated embryos is consistent with a model in which reception of a repulsive signal from the midline, which prevents longitudinal axons from following commissural pathways, was blocked by inhibition of TK activity. Since pCC extended a collateral branch across the midline in the presence of HA even though its primary growth cone had already chosen an ipsilateral pathway, the repulsive signal may be continuously required to keep longitudinal axons from crossing the midline.

What biochemical mechanisms could be involved in the transmission of this putative midline repulsive signal? Recent genetic results in Drosophila have provided important insights about repulsive signals from the midline, and these may be relevant to our results. The roundabout (robo) mutant has a phenotype in which many longitudinal axons are rerouted across the midline, resulting in widened commissures and reduced longitudinal tracts (Seeger et al., 1993). Longitudinal axons in robo embryos are observed to cross the midline, and commissural axons cross multiple times. The medial FasII pathway, which contains the pCC axon, follows a characteristic looping trajectory in robo mutants, forming circles or whorls around the midline. The pCC growth cone, which can be visualized with anti-FasII early in development, crosses the midline in robo mutants and fasciculates with its contralateral homolog (Kidd et al., 1998b). These phenotypes seem strikingly similar to those we observe in HA-treated grasshopper embryos (Figs 4, 5).

Robo is a transmembrane protein with immunoglobulin-like domains and fibronectin type III repeats (Kidd et al., 1998a). It has been proposed to function as a 'gatekeeper' that keeps longitudinal axons away from the midline and prevents midline recrossing by commissural axons. pCC and other longitudinal connective neurons express high levels of Robo. Interestingly, although the cytoplasmic domains of human, fly and nematode Robo proteins are greatly divergent, all of them contain a conserved tyrosine-containing motif, PXPYATT, at a similar position (Kidd et al., 1998b). This suggests that Robo signal transduction could involve phosphorylation of this tyrosine, followed by interaction of phosphorylated Robo with a PYbinding adapter protein. In grasshopper embryos, HA might inhibit a TK that normally phosphorylates Robo, so that Robo can no longer exert its gatekeeper function.

The other effect on guidance produced by TK inhibitors was the growth of additional axons out of the CNS in the SN root. This phenotype was seen in embryos treated with HA, GNS, or tyrphostin B42, while midline crossing by longitudinal axons was seen only in HA-treated embryos. About 27 motor axons normally exit the CNS via the SN root pathway. In inhibitor-treated embryos, however, approximately 88 
additional interneurons now also sent their axons out of the CNS in the SN root (Fig. 6). For both HA and GNS, the above phenotypes were accompanied by a decrease in the number of axons in the longitudinal connective (Fig. 4D-F). Thus, axons from the longitudinal connective were being rerouted into the $\mathrm{SN}$ root in the TK-inhibited embryos. These results suggest that there might be a choice point at which these interneuronal axons could either grow along the connective or follow the SN root, and that TK activity was required to choose the connective pathway.

HA and GNS, the TK inhibitors used in our study, act by different mechanisms (for review see Lawrence and Niu, 1998). HA has been classified as a specific inhibitor of Src family TKs, but can inhibit a variety of other TKs as well (Fukazawa et al., 1991; Graber et al., 1992). GNS is considered to be a broad-spectrum TK inhibitor, affecting the activities of many receptor and nonreceptor tyrosine kinases in vitro, including Src, Yes, Lck, the PDGFR and the EGFR (Akiyama et al., 1987). Our results imply that HA and GNS affected tyrosine phosphorylation of one or more substrata whose activity is crucial for correct axon guidance. Since HA, GNS and tyrphostin B42 all caused excess axons to enter the SN root, they may all share a common biochemical target(s) that is relevant to this phenotype. HA may also have affected phosphorylation of an additional target(s) relevant to the midline crossing phenotype, which is generated only in response to this inhibitor.

In conclusion, we have described two specific axon guidance phenotypes that occur as a consequence of TK inhibition in grasshopper embryos. Since we have been able to produce these guidance alterations in vivo by injecting eggs with inhibitors, we can study their long-term consequences by examining late embryos or hatched grasshoppers derived from injected eggs. For example, it will be of interest to determine whether muscle innervation patterns are changed as a result of the massive rerouting of interneuronal axons into the periphery.

We thank Michael Bastiani, Hugo Bellen and Mark Perin for antibodies; Barry Condron, Haig Keshishian, Chand Desai, Qi Sun and Richard Baines for helpful discussions; Doris Koo for help with in vivo microinjections; Rosalind Young for advice on EM techniques; Anna Vlasek for advice on western blots and Gilles Laurent for the use of his grasshopper colony. We also thank Markus Friedrich, Benno Schindelholz and Rusty Lansford for comments on the manuscript. This work was supported by an NIH RO1 grant and a Human Frontiers Science Project grant to K. Z.; K. M. was supported by an NRSA postdoctoral fellowship.

\section{REFERENCES}

Akiyama, T., Ishida, J., Nakagawa, S., Ogawara, H., Watanabe, S., Itoh, N., Shibuya, M. and Fukami, Y. (1987). Genistein, a specific inhibitor of tyrosine-specific protein-kinases. J. Biol. Chem. 262, 5592-5595.

Bastiani, M. J., Decouet, H. G., Quinn, J. M. A., Karlstrom, R. O., Kotrla, K., Goodman, C. S. and Ball, E. E. (1992). Position-specific expression of the Annulin protein during grasshopper embryogenesis. Dev. Biol. 154, 129-142.

Bastiani, M. J., Dulac, S. and Goodman, C. S. (1986). Guidance of neuronal growth cones in the grasshopper embryo.1. recognition of a specific axonal pathway by the pCC neuron. J. Neurosci. 6, 3518-3531.

Bastiani, M. J., Harrelson, A. L., Snow, P. M. and Goodman, C. S. (1987). Expression of fasciclin-I and fasciclin-II glycoproteins on subsets of axon pathways during neuronal development in the grasshopper. Cell 48, 745755.

Bastiani, M. J., Raper, J. A. and Goodman, C. S. (1984). Pathfinding by neuronal growth cones in grasshopper embryos.3. selective affinity of the ggrowth cone for the P-cells within the A/P fascicle. J. Neurosci. 4, 23112328.

Bentley, D., Keshishian, H., Shankland, M. and Toroian-Raymond, A. (1979). Quantitative staging of embryonic development of the grasshopper, Schistocerca nitens. J. Embryol. Exp. Morph. 54, 47-74.

Bixby, J. L. and Jhabvala, P. (1992). Inhibition of tyrosine phosphorylation potentiates substrate-induced neurite growth. J. Neurobiol. 23, 468-480.

Bixby, J. L. and Jhabvala, P. (1993). Tyrosine phosphorylation in early embryonic growth cones. J. Neurosci. 13, 3421-3432.

Callahan, C. A., Muralidhar, M. G., Lundgren, S. E., Scully, A. L. and Thomas, J. B. (1995). Control of neuronal pathway selection by a Drosophila receptor protein-tyrosine kinase family member. Nature 376, 171-174.

Condron, B. G. and Zinn, K. (1994). The grasshopper median neuroblast is a multipotent progenitor cell that generates glia and neurons in distinct temporal phases. J. Neurosci. 14, 5766-5777.

Condron, B. G. and Zinn, K. (1997). Regulated neurite tension as a mechanism for determination of neuronal arbor geometries in-vivo. Curr. Biol. 7, 813-816.

Desai, C. J., Gindhart, J. G., Goldstein, L. S. B. and Zinn, K. (1996). Receptor tyrosine phosphatases are required for motor axon guidance in the Drosophila embryo. Cell 84, 599-609.

Desai, C. J., Krueger, N. X., Saito, H. and Zinn, K. (1997). Competition and cooperation among receptor tyrosine phosphatases control motoneuron growth cone guidance in Drosophila. Development 124, 1941-1952.

Doherty, P., Furness, J., Williams, E. J. and Walsh, F. S. (1994). Neurite outgrowth stimulated by the tyrosine kinase inhibitor herbimycin-A requires activation of tyrosine kinases and protein-kinase-c. J. Neurochem. 62, 21242131.

Fukazawa, H., Li, P. M., Yamamoto, C., Murakami, Y., Mizuno, S. and Uehara, Y. (1991). Specific-inhibition of cytoplasmic protein tyrosine kinases by herbimycin-A in vitro. Biochem. Pharm. 42, 1661-1671

Graber, M., June, C. H., Samelson, L. E. and Weiss, A. (1992). The protein tyrosine kinase inhibitor herbimycin-A, but not genistein, specifically inhibits signal transduction by the T-cell antigen receptor. Intl. Immun. 4, 1201-1210.

Henkemeyer, M., Orioli, D., Henderson, J. T., Saxton, T. M., Roder, J., Pawson, T. and Klein, R. (1996). Nuk controls pathfinding of commissural axons in the mammalian central-nervous-system. Cell 86, 35-46.

Kaufmann, N., Wills, Z. P. and Vanvactor, D. (1998). Drosophila Rac1 controls motor axon guidance. Development 125, 453-461.

Kidd, T., Brose, K., Mitchell, K. J., Fetter, R. D., Tessierlavigne, M., Goodman, C. S. and Tear, G. (1998a). Roundabout controls axon crossing of the CNS midline and defines a novel subfamily of evolutionarily conserved guidance receptors. Cell 92, 205-215.

Kidd, T., Russell, C., Goodman, C. S. and Tear, G. (1998b). Dosagesensitive and complementary functions of Roundabout and Commissureless control axon crossing of the CNS midline. Neuron 20, 25-33.

Krueger, N. X., Vanvactor, D., Wan, H. I., Gelbart, W. M., Goodman, C. S. and Saito, H. (1996). The transmembrane tyrosine phosphatase dlar controls motor axon guidance in Drosophila. Cell 84, 611-622.

Landgraf, M., Bossing, T., Technau, G. M. and Bate, M. (1997). The origin, location, and projections of the embryonic abdominal motor-neurons of Drosophila. J. Neurosci. 17, 9642-9655.

Lawrence, D. S. and Niu, J. K. (1998). Protein-kinase inhibitors - the tyrosine-specific protein-kinases. Pharm. Thera. 77, 81-114.

Littleton, J. T., Bellen, H. J. and Perin, M. S. (1993). Expression of synaptotagmin in Drosophila reveals transport and localization of synaptic vesicles to the synapse. Development 118, 1077-1088.

Lowell, C. A. and Soriano, P. (1996). Knockouts of src-family kinases - stiff bones, wimpy T-cells, and bad memories. Genes Dev. 10, 1845-1857.

Luo, L. Q., Liao, Y. J., Jan, L. Y. and Jan, Y. N. (1994). Distinct morphogenetic functions of similar small Gtpases - Drosophila Drac1 is involved in axonal outgrowth and myoblast fusion. Genes Dev. 8, 17871802.

Meier, T., Chabaud, F. and Reichert, H. (1991). Homologous patterns in the embryonic-development of the peripheral nervous-system in the grasshopper Schistocerca-gregaria and the fly Drosophila-melanogaster. Development 112, 241-253.

Miller, D. R., Lee, G. M. and Maness, P. F. (1993). Increased neurite 
outgrowth induced by inhibition of protein-tyrosine kinase-activity in PC12 pheochromocytoma cells. J. Neurochem. 60, 2134-2144.

Miyamoto, S., Teramoto, H., Coso, O. A., Gutkind, J. S., Burbelo, P. D., Akiyama, S. K. and Yamada, K. M. (1995). Integrin function - molecular hierarchies of cytoskeletal and signaling molecules. J. Cell Biol. 131, 791805.

Myers, P. Z. and Bastiani, M. J. (1993). Growth cone dynamics during the migration of an identified commissural growth cone. J. Neurosci. 13, 127143.

Orioli, D., Henkemeyer, M., Lemke, G., Klein, R. and Pawson, T. (1996). Sek4 and Nuk receptors cooperate in guidance of commissural axons and in palate formation. EMBO J. 15, 6035-6049.

Patel, N. H., Ball, E. E. and Goodman, C. S. (1992). Changing-role of Evenskipped during the evolution of insect pattern-formation. Nature 357, 339342.

Patel, N. H., Martinblanco, E., Coleman, K. G., Poole, S. J., Ellis, M. C., Kornberg, T. B. and Goodman, C. S. (1989). Expression of Engrailed proteins in arthropods, annelids, and chordates. Cell 58, 955-968.

Perin, M. S., Johnston, P. A., Ozcelik, T., Jahn, Francke, U. and Sudhof, T. C. (1991). Structural and functional conservation of Synaptotagmin (P65) in Drosophila and humans. J. Biol. Chem. 266, 615-622.

Petrey, D., Buster, D., Donato, K. K. and Anderson, H. (1989). Injection of antibodies into grasshopper eggs as a method for studying embryonicdevelopment. Dev. Growth Different. 31, 299-305.

Seaver, E. C., Karlstrom, R. O. and Bastiani, M. J. (1991). The restricted spatial and temporal expression of a nervous-system-specific antigen involved in axon outgrowth during development of the grasshopper. Development 111, 881-893.

Seeger, M., Tear, G., Ferresmarco, D. and Goodman, C. S. (1993). Mutations affecting growth cone guidance in Drosophila - genes necessary for guidance toward or away from the midline. Neuron 10, 409-426.

Sink, H. and Whitington, P. M. (1991). Location and connectivity of abdominal motoneurons in the embryo and larva of Drosophilamelanogaster. J. Neurobiol. 22, 298-311.

Speicher, S., Garciaalonso, L., Carmena, A., Martinbermudo, M. D. Delaescalera, S. and Jimenez, F. (1998). Neurotactin functions in concert with other identified CAMs in growth cone guidance in Drosophila. Neuron 20, 221-233.

van Essen, D. C. V. (1997). A tension-based theory of morphogenesis and compact wiring in the central nervous system. Nature 385, 313-318.

Von Bernhardi, R. and Bastiani, M. J. (1995). Requirement of RNAsynthesis for pathfinding by growing axons. J. Comp. Neurol. 357, 52-64.

Whitington, P. M. (1989). The early development of motor axon pathways in the locust embryo - the establishment of the segmental nerves in the thoracic ganglia. Development 105, 715-721.

Worley, T. and Holt, C. (1996). Inhibition of protein-tyrosine kinases impairs axon extension in the embryonic optic tract. J. Neurosci. 16, 2294-2306.

Xie, F. K., Meier, T. and Reichert, H. (1992). Embryonic-development of muscle patterns in the body wall of the grasshopper. Roux's Arch. Dev. Biol. 201, 301-311. 